\title{
Erratum: Disorder Criterion and Explicit Solution for the Disc Random Packing Problem [Phys. Rev. Lett. 127, 118002 (2021)]
}

\author{
Raphael Blumenfeldœ
}

(Q) (Received 20 November 2021; published 15 December 2021)

DOI: 10.1103/PhysRevLett.127.259901

In my recent Letter [1], the range of values of $\theta_{2}$, shown in Eq. (5), was miscalculated. The limits are

$$
\begin{aligned}
\frac{\pi}{6}<\theta_{2}<\theta_{1}+\frac{\pi}{6} & \text { for } \frac{\pi}{6}<\theta_{1}<\frac{\pi}{3}, \\
\theta_{1}-\frac{\pi}{6}<\theta_{2}<\frac{5 \pi}{6}-\theta_{1} & \text { for } \frac{\pi}{3}<\theta_{1}<\frac{\pi}{2} .
\end{aligned}
$$

This affects slightly the limits of the integrals in Eq. (6), yielding $\bar{S}_{5}=1.549782$ 5. Consequently, the following values are modified very slightly: $\phi_{\mathrm{RCP}}=0.852514$, which is lower by $1.1 \times 10^{-5}$ than the originally published value, and $\phi_{c s}=0.830737$, which is lower by $2.3 \times 10^{-4}$ than the originally published value. All other calculations are unaffected.

Thanks go to Dr. Himantha Cooray for spotting a potential problem with the original limits of Eq. (5) and for checking the revised calculation.

[1] R. Blumenfeld, Phys. Rev. Lett. 127, 118002 (2021). 